\title{
The Use of Broncho-Alveolar or Gastric Lavage for the Diagnosis of Pulmonary Tuberculosis in Ouagadougou, Burkina Faso
}

\author{
Souba Diande ${ }^{1,2}$, Arend H. J. Kolk ${ }^{3}$, Isidore Moyenga ${ }^{2}$, Eric Zoungrana ${ }^{2}$, Adama Ouédraogo ${ }^{2}$, Arnaud Konseimbo ${ }^{2}$, \\ Léon T Sawadogo, Lassana Sangaré ${ }^{4,5}$ \\ 1. Laboratoire National de Référence des mycobactéries, Ouagadougou, Burkina Faso \\ 2. Coordination du Programme National Tuberculose (PNT), Ouagadougou, Burkina Faso \\ 3. Analytical Chemistry \& Forensic Science, University of Amsterdam, 1098XH Amsterdam, The Netherlands \\ 4. Service de Bactériologie-Virologie, Centre Hospitalière Universitaire Yalgado Ouédraogo, Ouagadougou \\ 5. Unité de Formation et de Recherches Sciences de la Santé, Université de Ouagadougou
}

\begin{abstract}
Background: the national and international guidelines of the WHO and the IUALTD recommend priority sputum smears for diagnosis of pulmonary tuberculosis. We studied the use and diagnostic yield of sputum, broncho-alveolar and gastric lavage (BAL, $\mathrm{GL}$ ) in the reference national mycobacteria Laboratory (NRL) (Burkina Faso) to see if clinicians follow the recommendations in the prescription of specimens. Methods: We analyzed by microscopy all samples sent to the RNL from 8 public and 6 private health care facilities in 2010. Results: 2638 patients were included. We have reported on 2414 early morning sputum samples, BAL from 191 patients and gastric lavage from 33. There were significant differences in the type of sample submitted per institution. Sputum formed $95 \%$ of the 862 samples from one hospital, whereas $78 \%$ of the 223 samples submitted by one private clinic were BAL samples. No sputum samples were submitted from these patients and in this clinic the diagnostic yield of BAL was just $4 \%$. The overall diagnostic yield for early morning sputum specimens was 12\% (290/2414), for BAL 3.7\% (7/191) and for GL 6\% (2/33). Conclusion: there was considerable variation in the diagnostic procedure used. Invasive methods are used at some sites in preference to safer and simpler means of obtaining material for diagnosis. Our review shows the need for constant vigilance and close adherence to internationally accepted guidelines for the diagnosis of pulmonary TB.
\end{abstract}

Key words: Acid-fast bacteria, gastric lavage, broncho-alveolar lavage.

\section{Introduction}

One third of the world's population is infected with tuberculosis (TB), which is a leading cause of death from infectious disease. The World Health Organization (WHO) estimated that in 2010 there were 8.8 million new TB patients and 1.45 million deaths from the disease, of which one third were due to $\mathrm{HIV}$-associated TB. In developing countries TB is a major cause of mortality in those infected by the human immunodeficiency virus (HIV) $[1,2]$.

Corresponding author: Diande Souba, $\mathrm{PhD}$, research fields: mycobacteria. E-mail: diandesouba@hotmail.com.
The definitive diagnosis of $\mathrm{TB}$ is based on the culture of Mycobacterium tuberculosis from clinical specimens, e.g., sputum, broncho-alveolar lavage (BAL), gastric lavage (GL). It takes two to eight weeks before the culture results are available and the technique requires trained and qualified staff and costly equipment.

Burkina Faso is a developing country with a population of 16 million and an estimated incidence of TB of 55 cases per 100,000 inhabitants [1]. Resources are limited. There is a National Tuberculosis Program (NTP) that has adopted the DOTS (Directly Observed Therapy Short-course) and the Stop TB global plan 
recommended by the WHO and the International Union against Tuberculosis and Lung Diseases (IUALTD). The key technique for the diagnosis of TB is the direct microscopic examination of relevant clinical samples after preparation and Ziehl-Neelsen staining for light microscopic detection or auramine staining for fluorescence microscopy of the bacteria [3, 4]. This permits the identification and treatment of those with the most dangerous form of pulmonary TB, since these patients may spread the disease in the community.

For diagnostic purposes, two samples of good quality sputum are requested from those with suspected respiratory tuberculosis: patients with nocturnal cough persisting for more than 2 weeks despite antibiotics. The National Tuberculosis Program recommends that when there is difficulty in obtaining sputum, particularly in children or HIV-positive patients, GL should be done [5]. In those patients where the index of suspicion is high despite two negative sputum smears, a BAL should be considered [6]. This however is an invasive procedure with the risks associated with any bronchoscopy of infection, pneumothorax and lung collapse, as well as the risk of potential contamination of the bronchoscope.

A brief review of our workload in 2010 showed considerable disparities in the use of sputum, BAL or GL samples for the diagnosis of pulmonary TB, and we decided to look at this in greater detail. We have reviewed the type of sample sent to our laboratory, the national reference mycobacteria laboratory for microscopy. Samples from 2638 patients were sent from 14 different health care facilities in Ouagadougou, the capital city of Burkina Faso. We have looked at the diagnostic yields of the different techniques and compared these with the experience of others in different countries. We have included samples from adults and from those aged less than 15 years old. It is relevant to note that a patient must pay $0.76 €$ for analysis of specimens by microscopy but eighty times this, $62 €$, for the BAL procedure and doctor's costs.
For patients receiving treatment, follow-up microscopy is free.

\section{Clinical Materials and Methods}

\subsection{Inclusion Criteria}

We have examined all clinical specimens sent for the detection of acid-fast bacilli (AFB) to the national reference mycobacteria Laboratory in Ouagadougou, between January and December 2010. These samples came from 2638 new patients with suspected pulmonary TB who were attending one of the 14 health care facilities (8 public and 6 private) in Ouagadougou that do not have their own facilities for Ziehl-Neelsen microscopy. Sources included the CNLAT, the University Hospital (Centre Hospitalier Universitaire Yalgado Ouédraogo), the infirmary of the House of Judgment and Correction of Ouagadougou - the local prison, 5 other public care units and 6 private Clinics/care units, identified as Private clinic 1, Private clinics/care units 2 to 6 . As well as details of the specimen type and microbiological data, we also recorded details of age, sex and location of the patient. Patients were analyzed separately by age: less than 15 years of age or older. Patients in whom AFB were identified by microscopy were offered treatment free of charge at CNLAT, the University hospital or at a care centre nearer their home.

\subsection{Clinical Samples}

The National Tuberculosis Programme recommends that 2 samples of sputum should be collected from each patient with suspected pulmonary TB: one "spot" and one early morning sample [5]. In 2010 we received 4828 viscous or mucopurulent sputum samples from 2414 patients with suspected TB. Since the early morning sample gives a higher diagnostic yield [5], we present the results here of the morning sputum smear (2414 samples). Gastric lavage was collected in 2 facilities, from 28 in-patients at the University hospital and 5 outpatients at Private clinic 1. Samples were collected using a 14 gauge nasogastric 
tube in the morning after the patient had fasted overnight. Two $20 \mathrm{ml}$ aliquots of isotonic saline were injected via the tube and retrieved by aspiration. Broncho-alveolar lavage specimens were submitted from 18 patients in the University hospital and 173 patients from Private clinic 1. Samples were collected via a bronchoscope inserted into the right bronchus intermedius, using xylocaïne as a local anesthetic. Fifty $\mathrm{ml}$ samples of sterile isotonic saline were used for each washing, up to a total of $200 \mathrm{ml}$, until a volume of 50 $\mathrm{ml}$ had been aspirated.

\subsection{Ethical Considerations}

Approval of an ethics committee was not necessary.

\subsection{Microscopic Examination}

Sputum sample were prepared as described by the WHO guidelines [3]. GL or BAL samples were centrifuged at 3,000 rpm $(1650 \mathrm{xg})$ for 15 minutes. The supernatant was discarded. The pellet was resuspended in $0.1 \mathrm{ml}$ isotonic saline and used to prepare smears for microscopy. All smears were treated with auramine staining and examined with fluorescence microscopy using a total magnification of 400 times $(10 \times 40)$. Any sample showing a positive result was then examined further. A separate portion of the same sample (sputum, GL or BAL) was then treated with Ziehl-Neelsen stain and examined with bright field microscopy $(10 \times 100$ magnification) as described by the WHO; the $\mathrm{ZN}$ results were scored according to the WHO guidelines [3].

No acid-fast bacteria (AFB) per 100 immersion fields: score negative, $\mathrm{ZN}$ -

1-9 AFB per 100 immersion fields: score scanty, $\mathrm{ZN} \pm$; > 10-99 AFB per 100 immersion fields: score 1+, ZN+; 1-10 AFB per immersion field: score 2+, $\mathrm{ZN}++$; More than 10 AFB per immersion field: score 3+ $\mathrm{ZN}+++$.

\subsection{Statistical Tests}

Data were analyzed using SPSS 15.0 (Statistical
Package for the Social Sciences, Chicago, IL, USA) for Windows. Statistical analysis was performed by the test of Mantel-Haenszel to explore the difference of lavage products (BAL $+\mathrm{GL})$ and sputum AFB smears positivity. Odds ratio $(\mathrm{OR})$ was indicated. $P<0.05$ was considered statistically significant.

\section{Results}

\subsection{Patient Characteristics}

The patient ages ranged from 1-87 years with a median age of 40 years in those 15 or over. Median age in children of 14 years or less was 8 years. Two-thirds (1644) of all the 2638 patients with suspected pulmonary TB in 2010 were male.

\subsection{Details of Clinical Samples}

We report here on samples from 2638 patients including: 2414 early morning sputum specimens, 191 BAL and $33 \mathrm{GL}$. Table 1 shows the distribution of the different types of samples over the two age groups ( $<$ 15 years and $\geq 15$ years) that were investigated for the presence of AFB in 2010. All Health units provided sputum samples from all age groups except the prison infirmary where all the patients were more than 15 years old. Samples were received from 153 children, the vast majority $123(80 \%)$ of which were sputum samples. Sputum was also the principal source in adults. The University Hospital submitted BAL from 15 patients and GL from 17 patients but $95 \%$ of samples were sputum. In contrast Private clinic 1 delivered 223 clinical samples, of which $78 \%$ came from BAL, only 45 samples $(20 \%)$ were sputum and $2 \%$ were gastric lavage. No sputum sample was submitted by Private clinic 1 from any of the 173 patients subjected to BAL.

\subsection{Diagnostic Yield}

Twelve per cent of the early morning sputum samples were Ziehl-Neelsen microscopy positive (see Table 2). Samples from 213 of these patients showed a score of 2-3+, indicating that they had a high risk of spreading the disease. The overall yield from BAL was 
Table 1 Specimens submitted by the different health care facilities.

\begin{tabular}{|c|c|c|c|c|c|c|}
\hline \multirow{2}{*}{ Health unit } & & & \multicolumn{3}{|c|}{ Number (\%) of Specimens } & \multirow[t]{2}{*}{ Total } \\
\hline & & & Sputum & BAL & GL & \\
\hline \multirow{3}{*}{ University Hospital $^{1}$} & \multirow{2}{*}{ Age group } & $<15$ years & $64(76)$ & $3(4)$ & $17(20)$ & 84 \\
\hline & & $\geq 15$ years & 752(97) & $15(2)$ & $11(1)$ & 778 \\
\hline & Total & All ages & $816(95)$ & $18(2)$ & $28(3)$ & 862 \\
\hline \multirow{3}{*}{ CNLAT $^{2}$} & \multirow{2}{*}{ Age group } & $<15$ years & 39 & $-{ }^{4}$ omit & - & 39 \\
\hline & & $\geq 15$ years & 786 & - & - & 784 \\
\hline & Total & All ages & 825 & - & - & 825 \\
\hline \multirow{3}{*}{ Private clinic 1} & \multirow{2}{*}{ Age group } & $<15$ years & $2(17)$ & $9(75)$ & $1(8)$ & 12 \\
\hline & & $\geq 15$ years & $43(20)$ & $164(78)$ & $4(2)$ & 211 \\
\hline & Total & All ages & $45(20)$ & $173(78)$ & $5(2)$ & 223 \\
\hline \multirow{3}{*}{ Private clinic/care unit 2-6 } & \multirow{2}{*}{ Age group } & $<15$ years & 10 & - & - & 10 \\
\hline & & $\geq 15$ years & 310 & - & - & 310 \\
\hline & Total & All ages & 320 & - & - & 320 \\
\hline \multirow{3}{*}{ Five other public care units } & \multirow{2}{*}{ Age group } & $<15$ years & 8 & - & - & 8 \\
\hline & & $\geq 15$ years & 241 & - & - & 241 \\
\hline & Total & All ages & 249 & - & - & 249 \\
\hline Prison infirmary & Age group & $\geq 15$ years & 161 & - & - & 161 \\
\hline
\end{tabular}

${ }^{\mathrm{C}}$ Centre Hospitalier Universitaire Yalgado Ouédraogo, ${ }^{2}$ National Center for Tuberculosis

low at $3.7 \%(7 / 191)$ but for gastric lavage it was a little higher at $6 \%(2 / 33)$.

\section{Discussion}

The national guidelines for the diagnosis of pulmonary TB are published in the technical National Tuberculosis Programme guide [5] that is available in all public and private health care facilities in Burkina Faso. As part of Round 4 of the Global Fund, all workers involved in the fight against TB are trained and subjected to re-training every 2 years. Thus doctors, nurses and other clinical staff working for TB health care providers are familiar with the symptoms of pulmonary TB such as night cough persisting for more than 2 weeks despite antibiotics, fever, chest pain and weight loss and with the implications of an abnormal chest X-ray. They are also aware that sputum smear microscopy is the simplest and cheapest technique for diagnosis. According to the WHO and IULTD recommendations, the use of BAL or sputum induction for the diagnosis of TB is only justified if the patient is unable to produce sufficient sputum for examination, or when 2-3 high quality early morning sputum samples have been smear negative and a high index of suspicion of pulmonary TB persists [7-12]. Gastric lavage is appropriate in children under 5 who are unable to produce sputum reliably [9, 13, 14]. Some centers have used induced sputum [10], naso-pharyngeal aspiration [15], BAL [16] or a combination of BAL and GL [17] in children with varying degrees of success. In our study sputum, samples gave the highest diagnostic yield of $12 \%$ (213 positive 290 positive in Table 2 , in 2414 early morning samples tested). In contrast gastric lavage had a yield of $6 \%$ and BAL of only $3.7 \%$. High 
Table 2 Comparison of the diagnostic yields of different techniques according to the centre involved in our study.

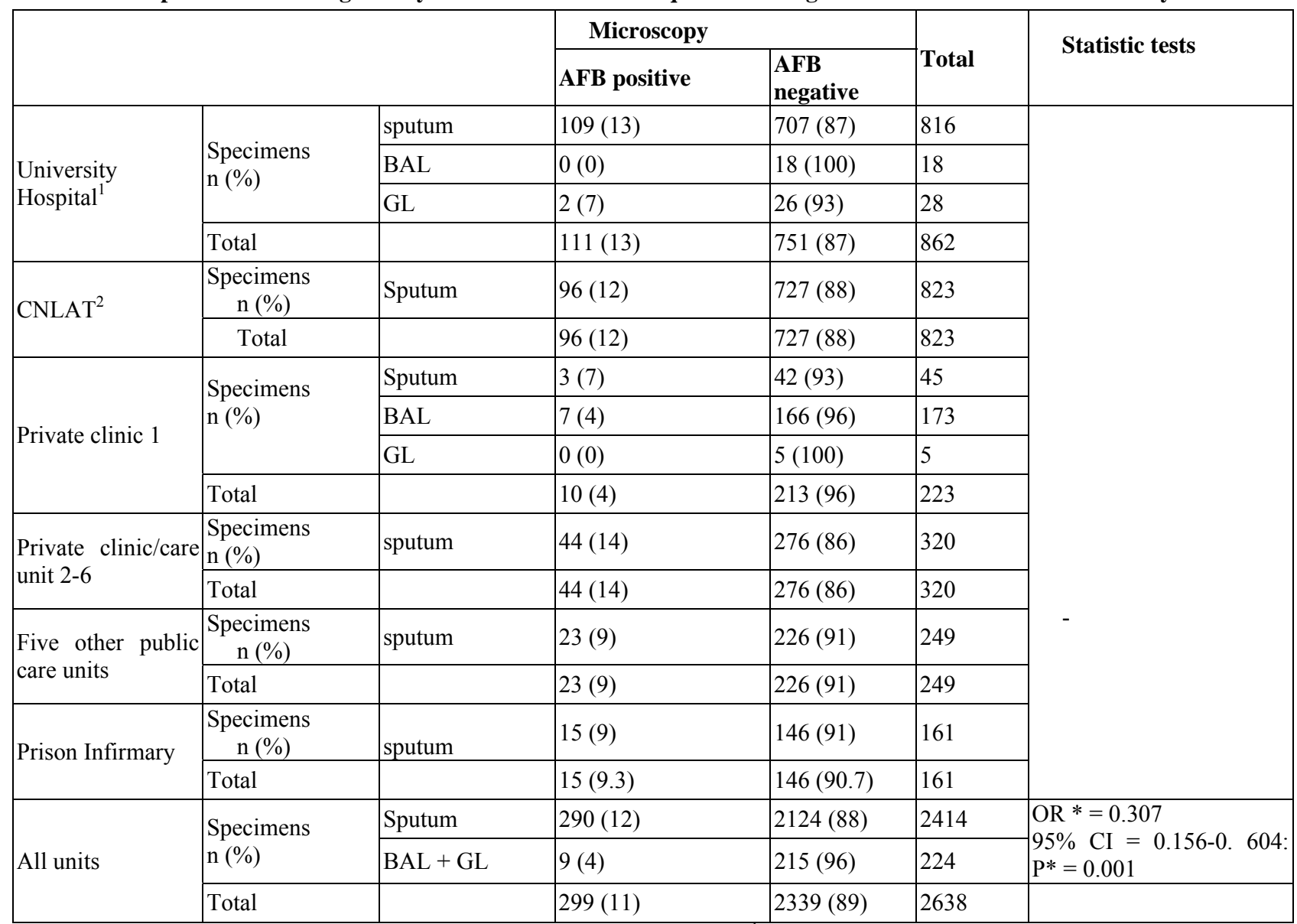

Abbreviations used: BAL, broncho alveolar lavage; GL, gastric lavage; ${ }^{1}$ Centre Hospitalier Universitaire Yalgado Ouédraogo; ${ }^{2}$ National Center for Tuberculosis; * = Mantel-Haenszel.

yields have been reported from gastric lavage, e.g., $28.3 \%$ in Brazilian children [13], 61.2\% in Turkish adults [11] and 90\% in Pakistani adults and children [18]. Low yields were also reported with gastric lavage from children in Zimbabwe, in Malawi and in South Africa (Table 3). When the results of GL are positive they need to be interpreted with caution because environmental AFB found in food, water and the soil may also be found in the stomach [21]. These will stain in the same way with Ziehl-Neelsen or auramine as $M$. tuberculosis. For this reason the WHO does not recommend GL for diagnostic purposes [21]. Where GL is the only source for a diagnostic specimen, we recommend culture or PCR for confirmation of $M$. tuberculosis complex infection if microscopy is positive.
It is reasonable to expect a higher yield than $3.7 \%$ for $\mathrm{BAL}$, a procedure that should be reserved for when simpler tests have failed and there is a strong suspicion of pulmonary TB. The Australian results with BAL with diagnostic yields of $37.8 \%$ and $34.3 \%$ in successive reports are notable in this regard, especially since each result reflects one year's experience [16, 22]. Other countries with disparate health care systems have also reported high yields, e.g., in Iran, in Switzerland and in Pakistan (Table 3). Interestingly, only $4.5 \%$ of BAL from adults in Malawi were positive [20]. In all of these studies patients were first required to produce sputum samples and they proceeded only to BAL or GL when these were negative but the index of suspicion based on clinical or radiological findings remained high. 
The very low yield from BAL specimens from Private clinic 1 is striking. It is worrying that no sputum was submitted for analysis from any of the patients subjected to BAL, contravening the national guidelines and the advice of WHO and IULTD. It seems unlikely that in a 12-month period not one of the 224 patients with suspected TB who had BAL was able to produce any sputum. Nine of the BAL samples came from children, where the role of BAL is controversial [25, 26]. BAL is an invasive procedure with a significant risk which should only be done when at least 2 high quality early morning sputum samples obtained on different days and examined in a laboratory with good quality controls have failed to give a diagnosis in a patient with strong clinical or radiological indications of TB. Since all personnel dealing with patients suspected of $\mathrm{TB}$ were subject to regular training updates, ignorance is not the explanation for the extravagant use of BAL at private clinic 1. In Burkina Faso, where the average monthly (the minimum guaranteed inter professional workers wages) [27] income is around $46.77 €$, the patient has to pay $0.76 €$ for laboratory analysis, $15.3 €$ doctor's costs and $46 €$ for the BAL procedure, a total of around $62 €$. Sputum examination costs the patient just $0.76 €$. It is unclear whether financial incentives played a role in the choice of BAL rather than sputum analysis at the Private clinic 1.

The NRL is a national reference laboratory for AFB microscopy and it participates in the international quality control program of microscopy organized by the WHO. The staff is well trained and is responsible for training and supervising the microscopists of the TB laboratory network throughout Burkina Faso. It is thus unlikely that we have missed cases of TB. We recommend that great care is taken in the choice of patients who are subjected to invasive diagnostic procedures, which should only be carried out when simpler measures have failed to give a diagnosis. Analyses such as the one presented here are valuable in allowing a health care system to detect providers that are outliers from the national norms and those who repeatedly fail to follow internationally agreed guidelines drawn up by experts, enabling interventions to be made for the benefit of patients.

\section{Conclusion}

We identified considerable variation in the different samples submitted for the diagnosis of pulmonary TB. Invasive methods are being used at some sites in preference to safer and simpler means of obtaining material for diagnosis. Our review of just one year's samples has shown the need for constant vigilance and close adherence to internationally accepted guidelines for the diagnosis of TB.

\section{Acknowledgments}

We thank the health professionals at the national reference mycobacteria laboratory in Ouagadougou for the technical work.

\section{References}

[1] WHO Report 2011 Global Tuberculosis Control, NLM classification: WF 300, 2011.

[2] R. Wood, G. Maartens and C. J. Lombard, Risk factors for developing tuberculosis in HIV-1 infected adults from communities with a low or very high incidence of tubercu-losis, J Acquir Immune Defic Syndr. 23 (2000) $75-80$.

[3] D. A. Enarson, H. L. Rieder, A. Thuridur and A. Trébucq, Support for tuberculosis: Union International against TB (5th ed.), 2000, p. 99.

[4] S. Diandé, G. Torrea and Ch. T. Ouattara et al., Diagnostic yield and grading of acid fast bacilli from sputum smears of HIV-infected and uninfected patients tuberculosis serial in routine microscopy laboratory in Ouagadougou, Burkina Faso, R Ci med biol Salvador 7 (2008) 288-293.

[5] Programme National Tuberculose, Burkina Faso. Guide technique de la lutte contre la tuberculose, 6ème éd, 2011, p. 148. (in French)

[6] K. Watanabe, Y. Inoue and T. Shimoda et al., Diagnostic usefulness of transbronchial aspiration and bronchial lavage for pulmonary tuberculosis, Kekkaku 65 (1990) 227-230.

[7] E. A. Khan and J. R. Starke, Diagnosis of tuberculosis in children: Increased need for better methods, Emerg Infect Dis. 4 (1995) 115-123. 


\section{Ouagadougou, Burkina Faso}

[8] T. McWilliams, A. U. Wells, S. Lindstrom, R. J. Cameron and E. Foskin, Induced sputum and bronchoscopy in the diagnosis of pulmonary tuberculosis, Thorax 57 (2002) $1010-1014$

[9] W. Okutan, Z. Kartaloğlu and E. Kılıc et al., Diagnosis Diagnostic? Contribution of gastric and bronchial washing examinations in cases suggestive of pulmonary tuberculosis, Yonsei Med J. 44 (2003) 242-248.

[10] H. J. Zar, D. Hanslo, P. Apolles, G. Swingler and G. Hussey, Induced sputum versus gastric lavage for microbiological confirmation of pulmonary tuberculosis in infants and young children: A prospective study, Lancet 365 (2005) 130-134.

[11] D. Saka, H. Celalettin and M. Ogretensoy, The diagnostic role of gastric aspiration in cases without sputum and in smear-negative patients with suspected pulmonary tuberculosis, Turk Resp J. 7 (2006) 124-128.

[12] M. Brown, H. Varia, P. Bassett, R. N. Davidson, R. Wall and G. Pasvol, Prospective study of sputum induction, gastric washing, and broncho-alveolar lavage for the diagnosis of pulmonary tuberculosis in patients who are unable to expectorate, Clin Infect Dis. 44 (2007) 1415-1420.

[13] E. L. N. Maciel, R. Dietze and R. P. Lyrio et al., Accuracy of inpatient and outpatient gastric lavage in the diagnosis of pulmonary tuberculosis in children, J Bras Pneumol. 34 (2008) 404-411.

[14] N. Al-Aghbari, N. Al-Sonboli and M. A. Yassin et al., Multiple sampling in one day to optimize smear microscopy in children with tuberculosis in Yemen, PLoS ONE 4 (2009) e5140.

[15] S. Owens, I. E. Abdel-Rahman, S. Balyejusa, P. Musoke, R. P. D. Cooke, C. M. Parry and J. B. S. Coulter, Nasopharyngeal aspiration for diagnosis of pulmonary tuberculosis, Arch Dis Child. 92 (2007) 693-696.

[16] R. Lumb, I. Bastian, Ch. Gilpin, P. Jelfs, T. Keehner and A. Sievers, Tuberculosis in Australia: Bacteriologically confirmed cases and drug resistance, 2005, A report of the Australian Mycobacterium Reference Laboratory Network, ILC 31 (2007) 80-86.

[17] P. R. Menon, R. Lodha, U. Singh and S. K. Kabra, A prospective assessment of the role of bronchoscopy and broncho-alveolar lavage in evaluation of children with pulmonary tuberculosis, J Trop Pediatr. 105 (2010) 1-5.
[18] N. Rizvi, N. A. Rao and M. Hussain, Yield of gastric lavage and bronchial washings in pulmonary tuberculosis, Int J Singapore Lung Dis. 4 (2000) 147-151.

[19] I. Garay, Clinical presentation of pulmonary tuberculosis in under $10 \mathrm{~s}$ and differences in AIDS-related cases: A cohort study of 115 patients, Trop Doct. 27 (1997) 139-142.

[20] D. J. Bell, R. Dacombe and S. M. Graham et al., Simple measures are as effective as invasive techniques in the diagnosis of pulmonary tuberculosis in Malawi, Int $\mathrm{J}$ Tuberc Lung Dis. 13 (2009) 99-104.

[21] World Health Organization, Laboratory Services in Tuberculosis Control, Microscopy part II; WHO/TB/98, pp. 258-263

[22] R. Lumb, I. Bastian and D. Dawson et al., Tuberculosis in Australia: bacteriologically confirmed cases and drug resistance, 2001, A report of the Australian Mycobacterium Reference Laboratory Network, ILC 27 (2003) 173-180.

[23] A. D. Khosravi, M. Mehdinejad, A. Mozzafari and M. Hashemzadeh, Study of sputum and bronchoscopic lavage for acid fast bacilli in patients with pulmonary infections, Afr J Microbiol Res. 4 (2010) 2414-2417.

[24] C. Tueller, P. N. Chhajed, C. Buitrago-Tellez, R. Frei, M. Frey and M. Tamm, Value of smear and PCR in broncho-alveolar lavage fluid in culture positive pulmonary tuberculosis, Eur Respir J. 26 (2005) 767-772.

[25] D. L. Abadco and P. Steiner, Gastric washing is better than bronchoalveolar lavage for isolation of Mycobacterium tuberculosis in childhood tuberculosis, Pediatr Infect Dis J. 11 (1992) 735-738.

[26] S. Chan, D. L. Badco and P. Steiner, Role of flexible fiber optic bronchoscopy in the diagnosis of childhood endobronchial tuberculosis, Pediatr Infect Dis J 13 (1994) 506-509.

[27] Burkina Faso, Salaire minimum interprofessionnel garanti. Décret $n^{\circ}$ 2006-655/PRES/PM du 29 décembre 2006, available online at: http://www.Droit-Afrique.com. (French)

[28] M. Rahbar, and M. Hajia, Value of gastric lavage for diagnosis of pulmonary tuberculosis, Pak J Med Sci. 23 (2007) 51-53. 